\title{
HIV Epidemic - The Importance of Sexual Education and Aiding Programs
}

\author{
Correa-Amorim LS ${ }^{1,2 *}$, Moncao-Meireles, AB ${ }^{1}$, Cine-Santos CC ${ }^{1}$, Barros, CS $^{1}$, Oliveira MC ${ }^{1,2}$ and Paixeo ICNP ${ }^{1,2}$ \\ ${ }^{1}$ Marine Biotechnology and Molecular Virology Laboratory, Department of Cellular and Molecular Biology, Biology Institute, Brazil \\ ${ }^{2}$ Department of Biology Institute, Brazil
}

*Corresponding author: Correa-Amorim LS, Department of Cellular and Molecular Biology, Biology Institute, Fluminense Federal University - Niterói Rio de Janeiro, Brazil.

Received Date: December 31, 2019

Published Date: January 30, 2020

\begin{abstract}
Despite all global efforts against the HIV/AIDS virus, it is alarming the high number of new infections episodes and unaware people regarding their serology, putting at risk one of the main goals of the WHO 90-90-90 program on the epidemic's control and eradication. In spite of the elevated number of countries that present systems of supporting and drawing seropositive individuals, one of the most significant concerns nowadays is, still, the transmission of HIV. PrEP, Pre-Exposure Prophylaxis is helping, considerably, in decreasing the cases of new infections. However, it also warns about the resurgence and growing of other Sexually Transmitted Infections (STI's).Which only makes us recognize sexual education as the central pillar, on raising population awareness about safe sexual practices, as well as predictors and risk factors, and acknowledge that the lack of a solid educational basis facilitates the practice of unsafe sex and increases the chances of exposing the person to HIV throughout life.
\end{abstract}

Keywords: HIV; Sexual education; Sexual risk factors; PrEP

\section{Introduction}

Retrovirus, nowadays recognized as HIV or Human Immunodeficiency Virus, was isolated only in 1983.Even ifthe Acquired Immune Deficiency Syndrome (AIDS) was originally acknowledged in 1981 when the number of opportunistic infections increased among young homosexuals. Since its first identification, HIV has been thoroughly studied. But it was only in 1985, that it was possible to develop licensed serology tests capable of detecting the virus infection. It is estimated that in over thirty years of HIV pandemic, the virus has been responsible for the infection of approximately 60 million people, and more than 25 million deaths, around the world [1-3].

Although the World Health Organization (WHO) had underestimated the pandemic in the past, since 1987, the organization has released a special program considering HIV named Global AIDS Plan (GAP). Hence, it has been observed a considerable advance in the treatment and control of the disease. The Nucleoside Reverse Transcriptase Inhibitors (NRTIs), as AZT and DDI were the first drugs available to treat the HIV infection. The efforts to contain the HIV pandemic continued after the 1990's decade. Due to the incomes generated by GPA and the significant number of donating countries, many underdeveloped nations were able to establish national controlling programs of HIV-AIDS infection. Their programs proved to be very efficient at that time, considering many countries were successful reverting epidemics or simply avoiding the worsening of the disease. However, the efforts were not enough, culminating in the closing of GPA's initiative and its substitution for the United Nations program UNAIDS [4,5].

In 1996 the Highly Active Antiretroviral Therapy (HAART) was introduced in the treatment of HIV. This therapy consists of the blending of three antiretroviral that focuses on at least two different molecular targets. This therapy suppresses viral replication drastically and also reduces its quantity, to levels lower than the detected limits established in the most sensible laboratory essays. Resulting in significant reconstitution of the immunologic system [6]. In 2003 WHO conceded the compulsory licensing of drugs in national emergencies, which enabled the importing of generic medicines by underdeveloped countries, and in some cases, as in Brazil, their production [5,7]. 
Since the isolation and presentation of HIV, many studies focused in its morphology. After it became clear that AIDS was caused by two distinct types of HIV, later nominated HIV-1 and HIV-2. Numerous HIV-1 strains are globally disseminated making clear the heterogeneity exhibited among them. Those two viral types present similarities in their morphology and tropism due to their lymphocytes $\mathrm{T} \mathrm{CD} 4+$, nevertheless, they are genetically distinguished [2].

\section{Epidemiology}

The HIV epidemic is responsible for approximately 1.5 million deaths around the world in 2017 [8], HIV-1 is considered the main causative agent of AIDS. UNAIDS published data of 2018, demonstrates that in the year 2017, 36 million people [30,8 42,9 million] were infected with HIV. Two types of HIV have been identified, type 1 and type 2, (HIV-1 and HIV-2) classified in groups and subtypes according to their distinct geographic distribution and origins [9]. HIV-1 isolated in 1983 [10], is widely distributed worldwide. While HIV-2 isolated in 1986 [11], can be found in Western Africa and some regions of Europe [12].

The strains of HIV-1 are organized in four different groups based on the viral genome, named: M (main), O (outlier), N (nom M or 0 ) and $\mathrm{P}$ (putative) $[13,14]$. The "M" group exhibits a significant number on a global scale responsible for $95 \%$ of infections by HIV1 [15]. The predominance of the other groups is extremely inferior, limited to Western Central Africa [16,17].

Divided into 9 subtypes, A, B, C, D, F, G, H, J and K, the M group has been evenly disseminated around the world. One example of that extensive dispersion is the subgroup B, the most prevalent form present in the American Continent and Europe yet considered rare in Africa [18]. Nowadays, the most common subtype of HIV1 in the world is subtype $C$ portraying the most prominent rate of infections (50\%), followed by subtype A (12\%) and subtype B (10\%) [19,20].

Today it is estimated that 40 million people in the world are living with HIV [21], experiencing social, personal and interpersonal traumas [22]. The annual global index of deaths regarding people who contracted HIV, according to UNAIDS, demonstrated a decline of 1.9 million deaths [1,4 - 2,7 million] in 2004 to a peak of 940 thousand deaths [670 thousand $-1,3$ million] in 2017. Since 2010 the mortality related to AIDS cases presented a decay of $34 \%$ [8]. The number of cases of HIV infection in the world continued to decline until 2017. Estimates indicate that there has been a significant decline in the number of new infections, with a decrease from 3.4 million [2,6 - 4,4 million] of new cases in 1996 to 1.8 million [1,4 - 2,4 million] in 2017 [8].

In Brazil since the manifestation of HIV in 1980 until 2011, it was recorded 608.203 registered occurrences of the disease manifestation. In 2010 alone were notified 34.218 new cases elevating the illness rate of incidence to 17.9 casesper 100.000 habitants. According to statistics in the last ten years there were changes on the sickness rates, where the South and the Southeast
Regions demonstrated a considerable reduction in the number of cases, while in other regions the increase was not expressive, except for the northern region where there was an increase from 7.0 to 20.6 cases [23].

Since 1980 when AIDS pandemic started until December 2017, it has been reported by the responsible organs in Brazil, more than 327 thousand deaths having as primary cause the HIV/AIDS. The higher percentage of deaths occurred in the Southeast Region, $58.9 \%$, followed by South and Northeast Regions both with $17.7 \%$ and $13.3 \%$ respectively, leaving the last positions to Central West and North Regions with $5.2 \%$ and $4.9 \%$ respectively [23].

\section{Predictors of Sexual Risk}

Sexual risk predictors and converging strategies are important to recognize behavioral targets that may be susceptible to the risk of HIV infection. Those risk predictors are important for prevention programs, besides from helping on the recognition of infected individuals that may be at a moderate level of infection. HIV is mainly transmitted through sexual intercourse worldwide. The transmission rate of new HIV cases among youngsters in the United States unrelated to sexual intercourse is lower than 5\% [24]. Therefore, for an effective prevention against HIV, it is necessary to have clear communication on the implications of the disease and the risk behaviors that should be avoided [25]. The most common via of HIV transmission is through sexual relations, representing 99\% of new occurrences in some Latin America countries. Behavioral monitoring can help to better evaluate the HIV prevalence as well as other Sexually Transmitted Infections (STI's), contributing to the development of effective prevention initiatives [26].

At the beginning of the HIV epidemic, individuals that demonstrated higher levels of schooling, higher available income, better possibilities of locomotion, and more favorable chances of establishing a wide sexual network, including sex professionals, were more susceptive to contract the disease. Deeper conscientization, safer sexual practices and access to precautionary health services produced an alteration in the scene of the correlation between the formal years of education and HIV [27]. Studies have demonstrated that youngsters who abandon formal education showcase a larger chance of having a higher number of sexual partners throughout their lives. Hence, increasing the frequency of sexual relations and also the incidence of unsafe sex when compared to those who continued in school [28].

Those statistics only confirm the fact that education is extremely important. It shapes sexual behavior with the aid of several mechanisms, and helps transform the dominant values of human behavior, such as knowledge, mindset, and social media action, consequently generating improvement in the socioeconomic level [29]. Information about sexual risk predictors should be used to provide the population with a better understanding through transparent, specific and scientifically updated messages. Enlightening people about the risks of transmission and infection by HIV and also its prevention strategies [25]. 


\section{HIV Intervention/Prevention Programmes}

Public health strategies to prevent HIV infection include educational campaigns to promote safe sexual practices, to expand and facilitate HIV tests and serotyping, to ease access to men circumcision and promote the antiretroviral prescription to seropositive individuals to diminish the circulating viral load, thus minimizing the risks of transmission [30]. At the beginning of the 2000s, the World Health Organization (WHO) created an initiative so people from poor countries living with HIV, could have total access to the antiretroviral treatment until the end of 2005 [31].

The efforts to expand the treatment were based on scientific evidence which confirmed that the early treatment could prevent the disease and, consequently, the deaths it caused. It also presented a decrease on virus transmission and helped to reduce health expenses [31].

The increasing global necessity and its compromise in solving the AIDS epidemic brought the UN Programme on HIV/AIDS (UNAIDS) to establish in 2014, the 90-90-90 goals (32). Those purposes provide a vital infrastructure to guide the response to HIV and to continue monitoring the progress of the epidemic termination [33].

The UNAIDS 90-90-90 program consists of reaching $90 \%$ of people living with HIV (PVHIV) to acknowledge their infection, with $90 \%$ of them taking antiretroviral therapy (TARV), and $90 \%$ of those getting their viral load suppressed [33]. UNAIDS predicts that, if we reach those goals until 2020, we will be able to end the AIDS epidemic until 2030 [32].

The 90-90-90 program focus on enhancing and maintaining constant care from the diagnosis to the viral suppression on the HIV infected individuals. The proportion of the specification among diagnosis, treatment, and viral suppression is the central pillar in response to HIV. The program's goal explicitly emphasizes the relevance of developing an adequate treatment to everyone in need and implicitly attests the requirement for monitoring systems to measure its progress [31]. The collected information about continued care is restricted now and describing the local, national and global continuous care has presented difficulties. Considering that there are no standardized approaches, or they neglected to detail how estimations had been determined [31].

Despite all efforts to reach that goal, we are still affected by 1.8 million HIV new infections every year, which demonstrates that prevention strategies are imperative. Clinical trials have been demonstrating that the use of medicines, like Pre-Exposure Prophylaxis (PrEP), can help decrease the risk of HIV transmission when used with high adherence [34].

PrEP is composed of daily therapeutics, employed through oral co-formulation of Tenofovir Disoproxil Fumarate combined with Emtricitabine (TDF/FTC). This combination has demonstrated to be safe and effective in the prevention of HIV infection in many population groups [35]. Validating those efficiency estimates, the shielding effect produced after correcting daily administration, was higher than $90 \%[36,37]$. UNAIDS's goal is to reduce the number of new infections to less than 500.000 cases per year until 2020, combining access to different prevention methods, including PrEP [38].

The motivation to use PrEP antiretroviral as an agent of preventing transmission of HIV infection was initially based on researches using test animals and case studies with humans that use post-exposure prophylaxis [30].

Depending on the country, PrEP can be prescribed like other prophylaxis drugs (one pill a day) or as "on-demand" prophylaxis medicine, before and after the person presents unsafe sexual behavior (two pills before the sexual activity, and one pill 24 and 48 hours after the first medicine) [38].

The United States was the first country to authorize the use of PrEP as a continuous prophylaxis drug in 2012. Nevertheless, only in 2016, France permitted the usage of PrEP as continuous prophylaxis medicine "on-demand". France was also the first country to offer a full refund to users of PrEP, which was later adopted by other European countries [38]. The World Health Organization (WHO) advises the prescription to PrEP to all individuals at high risk of HIV infection, for example, any person who belongs to a population group with an HIV incidence rate superior to $3 / 100$ people a year [39]. Those recommendations include men that make oral sex on other men (HSH), transsexual women, heterosexual men and women that maintain contact with unknown serology partners or untreated HIV positive people [38].

The identification of suitable candidates to the requirements of PrEP needs a complete and periodic review of the patients' drug use and sexual practices standards, so the higher-risk patients may be identified and beneficiated by PrEP [30]. One of the factors that discourage PrEP prescription is mostly the concern about an increase of unsafe sexual behavior; incentive to unprotected sex practice that consequentially, increases the ISTs incidence due to supposed protection provided by preventive treatment [38].

According to some studies, PrEP volunteers exhibited seropositivity on the initial screening to syphilis, HHV-2, gonorrhea (oral, rectal and urethral) and chlamydia. The high-level presence of ISTs before and after PrEP treatment had started, illustrates the importance of a screening routine for ISTs amid those patients [30]. PrEP completely changed the situation of HIV infection prevention, especially to HSH. It is clear that combined with ISTs screening and their immediate treatment, PrEP will considerably contribute to a large-scale incidents reduction, in countries where the treatment is available [38]. Strategies on HIV prevention are applied worldwide and widely divulged. Among those strategies, prevention through education continues to be a key factor in intervention policies, on national and global levels [39,40].

\section{Conclusion}

HIV infection still produces a substantial impact on public health due to its alarming epidemiological numbers. Despite all 
WHO efforts, in association with the treatment centers scattered around the world, the infection by HIV still reachs thousands of people, which makes even harder to achieve the goals established by WHO until 2020.When $90 \%$ of the infected population will have consciousness of their serology and $90 \%$ of that group, will be under antiretroviral treatment, and $90 \%$ of those treated individuals, will be viral-suppressed.

One of the strategies to be used is the PrEP, which has been proving to be a highly effective tactic against HIV transmission. Many countries, including Brazil, employ that tool as a neutralization mechanism of new infections, although, it is disturbing the emergence of strains resistant to the medicines, once PrEP is often constituted of drugs that compose the cocktail.

With the usage of PrEP, many individuals feel protected, which makes them vulnerable to ISTs. Therefore, the use of PrEP only shall not be enough to reduce the number of new infections worldwide. Coupled to this tool, sexual education, as well as formal schooling, are vital pillars to control the HIV infection. Researches indicate that the lack of knowledge regarding the virus, just as the failings on people's education, seems to be key factors on the fight against one of the greatest epidemics of all time.

\section{Acknowledgment}

The authors thank the Brazilian funding agencies CNPq, CAPES, and FAPERJ.

\section{Conflicts of Interest}

No conflicts of interest.

\section{References}

1. Del Rio C (2017) The global HIV epidemic: What the pathologist needs to know. Seminars in diagnostic pathology 34(4): 314-317.

2. Sauter D, Kirchhoff F (2019) Key Viral Adaptations Preceding the AIDS Pandemic. Cell host \& microbe 25(1): 27-38.

3. Sharp PM, Hahn BH (2011) Origins of HIV and the AIDS pandemic. Cold Spring Harbor perspectives in medicine 1(1): a006841.

4. Merson MH, O Malley J, Serwadda D, Apisuk C (2008) The history and challenge of HIV prevention. Lancet (London, England). 372(9637): 475-88.

5. Jamison DT, Breman JG, Measham AR, Alleyne G, Claeson M, et al. (2006) Disease Control Priorities in Developing Countries. Second Edition (eds) Oxford University Press and The World Bank, p. 1401.

6. Arts EJ, Hazuda DJ (2012) HIV-1 antiretroviral drug therapy. Cold Spring Harbor perspectives in medicine. 2(4): a007161.

7. Brito AM, Castilho EA, Szwarcwald CL (2001) AIDS and HIV infection in Brazil: a multifaceted epidemic. Revista da Sociedade Brasileira de Medicina Tropical 34(2): 207-217.

8. UNAIDS. Global, AIDS update 2018 / UNAIDS. Report 2018.

9. Essex M (1999) Human immunodeficiency viruses in the developing world. Advances in virus research 53: 71-88.

10. Barre-Sinoussi F, Chermann JC, Rey F, Nugeyre MT, Chamaret S, et al. (1983) Isolation of a T-lymphotropic retrovirus from a patient at risk for acquired immune deficiency syndrome (AIDS). Science (New York, NY) 220(4599): 868-871.

11. Clavel F, Guyader M, Guetard D, Salle M, Montagnier L, et al. (1986) Molecular cloning and polymorphism of the human immune deficiency virus type 2. Nature 324(6098): 691-695.
12. Kanki PJ, Travers KU, S MB, Hsieh CC, Marlink RG, Gueye NA, et al. (1994) Slower heterosexual spread of HIV-2 than HIV-1. Lancet (London, England) 343(8903): 943-946.

13. Plantier JC, Leoz M, Dickerson JE, De Oliveira F, Cordonnier F, et al. (2009) A new human immunodeficiency virus derived from gorillas. Nature medicine 15(8): 871-872.

14. Simon F, Mauclere P, Roques P, Loussert-Ajaka I, Muller-Trutwin MC, et al. (1998) Identification of a new human immunodeficiency virus type 1 distinct from group M and group 0. Nature medicine 4(9): 1032-1037.

15. Sharp PM, Hahn BH (2008) AIDS: prehistory of HIV-1. Nature 455(7213): 605-606.

16. Peeters M, Gueye A, Mboup S, Bibollet-Ruche F, Ekaza E, et al. (1997) Geographical distribution of HIV-1 group 0 viruses in Africa. AIDS (London, England) 11(4): 493-798.

17. Vallari A, Holzmayer V, Harris B, Yamaguchi J, Ngansop C, et al. (2011) Confirmation of putative HIV-1 group P in Cameroon. Journal of virology 85(3): 1403-1407.

18. Gilbert MT, Rambaut A, Wlasiuk G, Spira TJ, Pitchenik AE, et al. (2007) The emergence of HIV/AIDS in the Americas and beyond. Proceedings of the National Academy of Sciences of the United States of America 104(47): 18566-18570.

19. Hemelaar J, Gouws E, Ghys PD, Osmanov S (2011) Global trends in molecular epidemiology of HIV-1 during 2000-2007. AIDS (London, England) 25(5): 679-689.

20. Taylor BS, Sobieszczyk ME, McCutchan FE, Hammer SM (2008) The challenge of HIV-1 subtype diversity. The New England journal of medicine 358(15): 1590-1602.

21. Jaspal R, Kennedy L, Tariq S (2018) Human Immunodeficiency Virus and Trans Women: A Literature Review. Transgender health 3(1): 239-250.

22. Dulin AJ, Dale SK, Earnshaw VA, Fava JL, Mugavero MJ, et al. (2018) Resilience and HIV: a review of the definition and study of resilience. AIDS Care 2019: 1-12.

23. Boletim E (2018) Boletim epidemiológico HIV/Aids 2018. 49(HIV / AIDS): 72.

24. Mustanski BS, Newcomb ME, Du Bois SN, Garcia SC, Grov C (2011) HIV in young men who have sex with men: a review of epidemiology, risk and protective factors, and interventions. Journal of sex research 48 (23): 218-53.

25. Hess KL, Hu X, Lansky A, Mermin J, Hall HI (2017) Lifetime risk of a diagnosis of HIV infection in the United States. Annals ofepidemiology 27(4): 238-243.

26. Stuardo Avila V, Fuentes Alburquenque M, Munoz R, Bustamante Lobos L, Faba A, et al. (2019) Prevalence and Risk Factors for HIV Infection in a Population of Homosexual, Bisexual, and Other Men Who Have Sex with Men in the Metropolitan Region of Chile: A Re-emerging Health Problem. AIDS and behavior.

27. Bunyasi EW, Coetzee DJ (2017) Relationship between socioeconomic status and HIV infection: findings from a survey in the Free State and Western Cape Provinces of South Africa. BMJ open 7(11): e016232.

28. Hargreaves JR, Morison LA, Kim JC, Bonell CP, Porter JD, et al. (2008) The association between school attendance, HIV infection and sexual behaviour among young people in rural South Africa. Journal of epidemiology and community health. 62(2): 113-119.

29. Jukes M, Simmons S, Bundy D (2008) Education and vulnerability: the role of schools in protecting young women and girls from HIV in southern Africa. AIDS (London, England). 22(Suppl 4): S41-S56.

30. Riddell JT, Amico KR, Mayer KH (2018) HIV Preexposure Prophylaxis: A Review. Jama 319(12):1261-1268.

31. Granich R, Gupta S, Hall I, Aberle-Grasse J, Hader S, et al. (2017) Status and methodology of publicly available national HIV care continua and 90-90-90 targets: A systematic review. PLoS medicine 14(4): e1002253.

32. Staveteig S, Croft TN, Kampa KT, Head SK (2017) Reaching the 'first 90': Gaps in coverage of HIV testing among people living with HIV in 16 African countries. PloS one 12(10): e0186316. 
33. Hakim AJ, MacDonald V, Hladik W, Zhao J, Burnett J, et al. (2018) Gaps and opportunities: measuring the key population cascade through surveys and services to guide the HIV response. Journal of the International AIDS Society. 21(Suppl 5): e25119.

34. Gibas KM, Van den Berg P, Powell VE, Krakower DS (2019) Drug Resistance During HIV Pre-Exposure Prophylaxis. Drugs 79(6): 609-619.

35. Fonner VA, Dalglish SL, Kennedy CE, Baggaley R, O Reilly KR, et al. (2016) Effectiveness and safety of oral HIV preexposure prophylaxis for all populations. AIDS (London, England). 30(12): 1973-1983.

36. Anderson PL, Glidden DV, Liu A, Buchbinder S, Lama JR, et al. (2012) Emtricitabine-tenofovir concentrations and pre-exposure prophylaxis efficacy in men who have sex with men. Science translational medicine 4(151): 151ra25.

37. Donnell D, Baeten JM, Bumpus NN, Brantley J, Bangsberg DR, et al. (2014) HIV protective efficacy and correlates of tenofovir blood concentrations in a clinical trial of PrEP for HIV prevention. Journal of acquired immune deficiency syndromes (1999). 66(3): 340-348.
38. Siguier M, Molina JM (2018) HIV preexposure prophylaxis: An essential, safe and effective prevention tool for sexual health. Medecineet maladies infectieuses 48(5): 318-326.

39. WHO Guidelines Approved by the Guidelines Review Committee. Guidelines for The Diagnosis, Prevention and Management of Cryptococcal Disease in HIV-Infected Adults, Adolescents and Children: Supplement to the 2016 Consolidated Guidelines on the Use of Antiretroviral Drugs for Treating and Preventing HIV Infection. Geneva: World Health Organization

40. (2018) World Health Organization 2018.

41. Adelekan M (2017) A critical review of the effectiveness of educational interventions applied in HIV/AIDS prevention. Patient education and counseling 100(Suppl 1): S11-S16. 